\title{
COMMENT
}

\section{"Challenges and opportunities in academic medicine" APS racism series: at the intersection of equity, science, and social justice}

\author{
Leslie R. Walker-Harding ${ }^{1}$, Clifford W. Bogue ${ }^{2}$, Karen D. Hendricks-Munoz ${ }^{3}$, Jean L. Raphael ${ }^{4}$ and Joseph L. Wright ${ }^{5,6}$
}

Pediatric Research (2020) 88:699-701; https://doi.org/10.1038/s41390-020-01132-8

\section{AT THE INTERSECTION OF EQUITY, SCIENCE, AND SOCIAL JUSTICE: CHALLENGES AND OPPORTUNITIES IN ACADEMIC MEDICINE}

Academic medicine represents a unique and necessary platform from which to address professional and societal inequities. Undergraduate medical education and postgraduate medical education environments are steeped in the longstanding structural and systemic tenets that plague society more broadly. Through transparent acknowledgement of the inherent challenges, and intentional leveraging of the talent and scientific imprimatur embedded in our academic health systems, we can make progress in this area.

Diversity and inclusion matters

Academic medicine has been characterized by Kantor as a discipline that "encompasses the traditional tripartite mission of educating the next generation of physicians and biomedical scientists, discovering the causes of and cures for disease, and advancing knowledge of patient care while caring for patients... highlighting the interrelationships among teaching, research and service.... ." In working to fulfill these missions, academic health centers and medical schools should be responsive to societal trends and to the evolving healthcare needs of American society. Diversity and inclusion are critical as academic health centers and medical schools attempt to respond to societal healthcare needs, especially for marginalized and vulnerable population groups. Diversity and inclusion are also important for the development of a cadre of leaders from underrepresented minorities in medicine (URiM). Research studies have demonstrated several clear benefits to diversity and inclusion in clinical medicine, higher education, and workforce development. Ultimately, the evidence makes clear that the highest level of excellence in medicine will be achieved when actions show diversity and inclusion matter. A few examples include:

- Historically URiM graduates from medical school are three times more likely to serve minority and underserved populations than non-URiM and white medical school graduates. ${ }^{2,3}$

- Racial and ethnic concordance between patients and physicians enhances patient's experiences with the perception of receiving better interpersonal care. ${ }^{4}$
- Diversity in educational settings encouraged faculty to include themes relating to diversity in their research and teaching and provided students with opportunities to address racial and multicultural issues in the classroom and extracurricular settings. $^{5}$

- Students who interacted with racially and ethnically diverse peers showed the greatest engagement in active thinking as well as growth in intellectual engagement, motivation, and academic skills. ${ }^{6,7}$

- Diverse researchers help broaden the health research agenda, and lead to greater contributions to science as measured by impact factor and citations.

\section{Defining implicit bias}

Implicit bias, also known as unconscious bias, is a social stereotype about certain groups of people that individuals form outside their own conscious awareness. Implicit bias is not limited to ethnicity and race-it includes unconscious prejudice against another person(s) age, gender, religion, weight, sexual orientation, or gender identity. Research has shown that implicit biases develop at an early age, typically emerging during middle childhood and have real effects on a person's behavior, such as hiring, mentoring others, and health equity. ${ }^{9,10}$ Importantly, while implicit biases exist in all people, even ones that are incompatible with a person's conscious values, they are malleable and an individual can take steps to recognize and minimize the impact of their implicit biases. $^{11,12}$

Structural inequities and systemic barriers

Despite a large evidence-base demonstrating the value of diversity in academic medicine, progress towards diversity has been marginal as only a small number of physicians underrepresented in medicine (URiM) serve as faculty in medical schools. In 2018, only $3.6 \%$ of full-time faculty were Black or African American and 5.5\% were Hispanic, Latino, or Spanish. ${ }^{13}$ These inequities are compounded by disparities in a promotion such that minority faculty receive tenure at lower rates relative to their white counterparts, even after controlling for years of service and measures of academic productivity. A number of systemic barriers may account for these inequities. Faculty promotion

\footnotetext{
${ }^{1}$ Department of Pediatrics, University of Washington School of Medicine, Seattle, WA, USA; ${ }^{2}$ Department of Pediatrics, Yale University School of Medicine, New Haven, CT, USA; ${ }^{3}$ Department of Pediatrics, Virginia Commonwealth University College of Medicine, Richmond, VA, USA; ${ }^{4}$ Department of Pediatrics, Baylor College of Medicine, Houston, TX, USA;

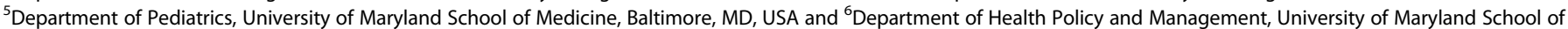
Public Health, College Park, MD, USA

Correspondence: Leslie R. Walker-Harding (leslie.walker-harding@seattlechildrens.org)
}

Received: 22 July 2020 Accepted: 28 July 2020

Published online: 17 September 2020 
committees and other decision-makers on career advancement may be relatively homogeneous, seek to be color blind, and not actively incorporate the experience and unique contributions of a URiM as an asset. Policies and procedures on what types of scholarship and individuals are valued by institutions can also be affected. URiM may experience professional isolation in that there are few individuals in their institutions who have similar life experiences as them. URiM faculty may have insufficient concordant role models, mentors, or sponsors. Lacking such a network may effectively exclude URiM faculty from informal information sharing, career advancement opportunities, and advocacy on their behalf when needed. URiM encounter implicit bias, discrimination, or overt racism from patients or colleagues. ${ }^{14,15}$ URiM also experience microaggressions, the insidious and ubiquitous nature of which make them difficult to identify and address. These various forms of bias may impact an individual's ability to function clinically and meet requisite scholarly milestones for career advancement and promotion. URiM often experiences the "minority tax", defined as the burden of extra responsibilities placed on URiM faculty to achieve diversity. ${ }^{16,17}$ The minority tax may manifest as diversity-related duties, such as committees, pipeline programs, recruitment events, and community service programs. While fulfilling publicly promoted values of the institution, such service may not be adequately supported, resourced, or recognized for promotion decisions, or even the desired area of scholarly focus for the URiM faculty member. As a result of all these structural factors, URiM faculty may report less satisfaction and engagement with their institution or colleagues, invest less in their professional development, discourage junior faculty URiM, or leave academic medicine altogether.

\section{Modification of educational experiences}

Our pediatric residency and fellowship training programs must reexamine what skills are required to successfully care for a US population of children who are majority non-white. If we are successful in our stated goal to increase diversity in the workforce and we have more African American, Latinx, and American Indian pediatric trainees, we will need to be simultaneously prepared to address how and in what context pediatric medicine is taught. In a recent study examining how Pediatric Departments felt they were doing with diversity and inclusion, the majority of them felt they were doing an excellent job; however, on further examination, many reported only very superficial actions like having a medical Spanish class for residents. ${ }^{18}$ With a renewed focus on becoming an antiracist society, it will be important to understand areas in residency education that will need change. Some examples are reframing the context research findings are reported and taught. In another recent study, half of the medical trainees surveyed had more than one false belief about Black patients, such as Black peoples' skin is thicker than white people, and that Black people were less sensitive to pain. ${ }^{19,20}$ Now that we know our trainees are likely to come to Pediatric residency with unchallenged, inaccurate notions about those they perceive as different from themselves, how are we going to reeducate them? How will we reeducate our academic teachers to address this and other health issues like racism? If we are successful, we will then create a group of researchers and clinicians and educators who will have the ability for the first time to finally improve the health and wellbeing of all of our nation's children.

Increasing a diverse pipeline: efforts must start in early childhood Without attention to fostering the pipeline and educational landscape, there will be continued inequities, not only in those receiving care as patients but also inequities in limiting workforce development and limiting opportunities for attaining postgraduate education and success in healthcare workforce leadership.
The first years of a child's life are rooted in early child developmental milestones and are also the foundations of later lifelong health disparities. For infants from underrepresented groups, these experiences form the foundation of later lifelong capabilities within educational, career, and socio-economic status. These early life experiences perpetuate continuing large-scale societal health disparities for those underrepresented in medicine groups, including African Americans, Latinx, and American Indians who form half of our nation's citizens and yet are represented at 9\% of the health workforce. ${ }^{4}$ Developing diversity in the educational areas of Science Technology Engineering and Medicine (STEM) and the healthcare workforce, to improve overall public health, has typically been structured at the health professions level in medical schools. Diversity inclusion and attention to bias and racism in educational resources and in the workforce can enhance curriculum education, acknowledge cultural differences as assets in helping to set institutional cultural norms, and decrease fear of cultural differences. Integrating STEM programs that occur at earlier stages of education with graduate medical education programs to have better continuity and develop a continuum of support for research and clinical and leadership careers in medicine is likely to improve the pipeline as well. Given the depth of nationally recognized educational thought leaders and experts in the American Pediatric Society (APS), we can make a significant contribution to educate, implement and become the "nation's pediatric educational compass." APS making a commitment to eliminate gender and racial and cultural inequalities in health education and healthcare will help to move the field forward.

\section{AUTHOR CONTRIBUTIONS}

L.R.W.-H. conceptualized the manuscript; J.L.W. developed the outline and content areas; L.R.W.-H, J.L.W., C.W.B., K.D.H.-M., and J.L.R. contributed content to the manuscript; L.R.W.-H and J.L.W. reviewed and revised the manuscript; and all authors reviewed and approved the revised manuscript as submitted and agreed to be accountable for all aspects of the work.

\section{ADDITIONAL INFORMATION}

Competing interests: The authors declare no competing interests.

Publisher's note Springer Nature remains neutral with regard to jurisdictional claims in published maps and institutional affiliations.

\section{REFERENCES}

1. Kanter, S. L. What is academic medicine? Acad. Med. 83, 205-206 (2008).

2. Saha, S. \& Shipman, S. A. The Rationale for Diversity in the Health Professions: $A$ Review of the Evidence (US Department of Health and Human Services, Health Resources Administration, Bureau of Health Professions, Rockville, 2006).

3. Saha, S. Taking diversity seriously: the merits of increasing minority representation in medicine. JAMA Intern. Med. 174, 291-292 (2013).

4. Smedley, B. D., Stith, A. Y. \& Nelson, A. R. (ed.). Unequal Treatment: Confronting Racial and Ethnic Disparities in Health Care. Institute of Medicine (US) Committee on Understanding and Eliminating Racial and Ethnic Disparities in Health Care (National Academies Press (US), Washington, 2003).

5. Milem, J. F., Chang, M. J. \& Antonio, A. L. Making Diversity Work on Campus: A Research-Based Perspective (Association of American Colleges and Universities, Washington, 2005).

6. Gurin, P. The compelling need for diversity in higher education: expert testimony in Gratz et al. v. Bollinger et al. Mich. J. Race Law 5, 363-425 (1999).

7. Gurin, P., Dey, E. L. \& Hurtado, G. G. Diversity and higher education: theory and impact on educational outcomes. Harv. Educ. Rev. 72, 330-66. (2002).

8. Valentine, H. A. \& Collins, F. S. National Institutes of Health addresses the science of diversity. Proc. Natl Acad. Sci. USA 112, 12240-12242 (2015).

9. Dore, R. A., Hoffman, K. M., Lillard, A. S. \& Trawalter, S. Children's racial bias in perceptions of others' pain. Br. J. Dev. Psychol. 32, 218-231 (2014).

10. Dasgupta, N. Implicit ingroup favoritism, outgroup favoritism, and their behavioral manifestations. Soc. Justice Res. 17, 143-169 (2004). 
"Challenges and opportunities in academic medicine" APS racism series: at... LR Walker-Harding et al.

11. Dasgupta, N. Implicit attitudes and beliefs adapt to situations: a decade of research on the malleability of implicit prejudice, stereotypes, and the selfconcept. Adv. Exp. Soc. Psychol. 47, 233-279 (2013).

12. Dasgupta, N. \& Greenwald, A. G. On the malleability of automatic attitudes: combating automatic prejudice with images of admired and disliked individuals. J. Pers. Soc. Psychol. 81, 800-814 (2001).

13. Association of American Medical Colleges Faculty Roster. Diversity in Medicine: Facts and Figures 2019. https://www.aamc.org/data-reports/workforce/interactivedata/figure-15-percentage-full-time-us-medical-school-faculty-race/ethnicity2018.

14. Ackerman-Barger, K., Boatright, D., Gonzalez-Colaso, R., Orozco, R. \& Latimore, D. Seeking inclusion excellence: understanding racial microaggressions as experienced by underrepresented medical and nursing students. Acad. Med. 95 758-763 (2020)
15. Dixon, G. et al. Factors that influence the choice of academic pediatrics by underrepresented minorities. Pediatrics.144 (2019).

16. Rodríguez, J. E., Campbell, K. M. \& Pololi, L. H. Addressing disparities in academic medicine: what of the minority tax? BMC Med. Educ. 15, 6 (2015).

17. Cyrus, K. D. A piece of my mind: medical education and the minority tax. JAMA 317, 1833-1834 (2017).

18. Mendoza, F. et al. Diversity and inclusion training in Pediatric Departments. Pediatrics 135, https://doi.org/10.1542/peds.2014-1653 (2015).

19. Hoffman, K. M., Trawalter, S., Axt, J. R. \& Oliver, M. N. Racial bias in pain assessment and treatment recommendations, and false beliefs about biological differences between blacks and whites. Proc. Natl Acad. Sci. USA 113, 4296-4301 (2016).

20. Villarosa, L. How false beliefs in physical racial difference still live in medicine today. 144, e20182759. https:/www.nytimes.com/interactive/2019/08/14/magazine/racialdifferences-doctors.html (2019). 